Мокрозуб В.Г.

\title{
ФУНКЦИОНАЛЬНАЯ И ПРОЦЕДУРНАЯ МОДЕЛИ ПРОЕКТИРОВАНИЯ ТЕХНОЛОГИЧЕСКОГО ОБОРУДОВАНИЯ
}

\begin{abstract}
Аннотация: Составлена функциональная модель (диаграмма) прочесса проектирования технологических аппаратов, включающая этапы структурного и параметрического синтеза аппарата и его элементов. На основании функциональной модели проектирования разработана процедурная модель, предназначенная для преобразование информационного потока, определенного техническим заданием, в информационный поток рабочего проекта. Техническое задание включает в себя множество функций проектируемого аппарата, условия взаимодействия аппарата с человеком, с рабочей и окружающей средой. Рабочий проект включает в себя чертежи, спецификации, расчеты и др. документы. Функциональная диаграмма составлена по методологии IDEFO. Процедурная модель представлена набором формальных выражений, преобразования входных информачионных поков в выходные. Прочедурная модель проектирования технологических аппаратов, отличается использованием информационно-логической модели проектируемого объекта, модели процессов, протекающих в аппарате, и модели технологии изготовления технического объекта. Разработанные функциональная и процедурная модели являются основой для создания интеллектуальных автоматизированных систем, позволяющих получать проектную документацию с минимальным участием человека. Ключевые слова: технологический аппарат, функциональная модель проектирования, процедурная модель, автоматизированное проектирование, интеллектуальные системы, математическое обеспечение, информачионная модель, гиперграф, структура изделия, рабочий проект
\end{abstract}

\section{Введение}

В проектировании технических объектов можно выделить два основных направления: поисковое и типовое. Результатом поискового проектирования является оригинальная конструкция, претендующая на получения патента. Результат типового проектирования - технический объект, собранный из типовых элементов. Далее речь пойдет о проектировании типовых технических объектах (технологического оборудования), например емкостные аппараты, кожухотрубчатые теплообменники и др.

Это оборудование предназначено для осуществления физических, химических или 
физико-химических процессов (химическая реакция, теплообмен без изменения агрегатного состояния, испарение, конденсация, кристаллизация, растворение, выпарка, ректификация, абсорбция, адсорбция, сепарация, фильтрация и др.), а также для хранения и перемещения различных веществ.

Технологическое оборудование состоит преимущественно из типовых элементов, что дает возможность создать информационную систему, которая позволит автоматизировать все стадии разработки проектной документации с минимальным участием человека $[1,2]$. Последнее требует наличия у автоматизированной информационной системы интеллектуальных свойств, например, знаний проектировщика, выраженных в форме продукционных правил [3,4].

Интеллектуальная информационная система автоматизированного проектирования технологического оборудования базируется на моделях процесса проектирования и проектируемого объекта, которые описывают процесс и объект, как на ранних, так и на заключительных стадиях создания проектной документации.

Для создания подобных моделей необходимо выявить:

- $\quad$ этапы проектирования технологического оборудования;

- $\quad$ информационные потки на входе и выходе каждого этапа;

- информационные потоки, связывающие различные этапы проектирования.

В первом разделе настоящей работы описывается функциональная модель процесса проектирования технологического оборудования, составленная по методологии IDEFO [5]. Во втором - процедурная модель процесса проектирования технологического оборудования, позволяющая по техническому заданию получить проектную документацию.

\section{Функциональная модель процесса проектирования технологического оборудования}

Процесс проектирования технологического оборудования состоит из следующих основных этапов [6,7]:

- $\quad$ определение структуры (концептуальное проектирование);

- технологические расчеты;

- разработка конструкции;

- разработка технологии изготовления;

- $\quad$ внесение изменений.

Исходные данные для проектирования технологического оборудования представлены в техническом задании (ТЗ), в котором отражены его функции, условия взаимодействия с окружающей и рабочей средой, ограничения на параметры

$$
T 3=\left\{L, F a, Q_{1}, Q_{2}, Q_{3}, Q_{4}\right\},
$$

где $L$ - основной размер оборудования оборудования (например, объем корпуса емкостного аппарата, поверхность теплообмена теплообменника, поверхность фильтрации фильтра и т. д.); 
$F a=\{f a\}-$ множество функций проектируемого оборудования;

$Q_{1}$ - условия взаимодействия аппарата с рабочей средой (давление, температура, коррозионные свойства и др.);

$Q_{2}$ - условия взаимодействия аппарата с окружающей средой (место установки, ветровые, снеговые нагрузки и. т.д.);

$Q_{3}$ - условия взаимодействия аппарата с человеком (требования к обслуживанию и безопасности);

$Q_{4}$ - дополнительные требования и ограничения (например, ограничение по габаритным размерам).

Функции аппарата $f a=(D, G, H)$,

где $D$ - указание действия, производимого аппаратом;

$G$ - указание объекта, на который направлено действие;

$H$ - указание особых условий и ограничений, при которых выполняется действие.

Результат проектирования - рабочий проект (РП).

$P \Pi=\left\{P \Pi_{k}\right\}, k=1,2 \ldots 9$,

где $Р \Pi_{1}$ - сборочный чертеж аппарата;

$P \Pi_{2}$ - сборочные чертежи отдельных частей аппарата;

$P \Pi_{3}$ - чертежи всех деталей аппарата;

$P \Pi_{4}$ - спецификации;

$P \Pi_{5}$ - паспорт аппарата;

$P \Pi_{6}$ - технологические расчеты процессов, протекающих в аппарате;

$P \Pi_{7}$ - расчет на прочность;

$P \Pi_{8}-$ руководство по эксплуатации;

$P \Pi_{9}$ - технологическая документация.

Для представления этапов процесса проектирования технологического оборудования и информационных потоков, присутствующих при проектировании, воспользуемся методологией IDEF0 [5].

Контекстная диаграмма верхнего уровня, отображающая связи объекта с окружающей средой, представлена на рисунке 1.

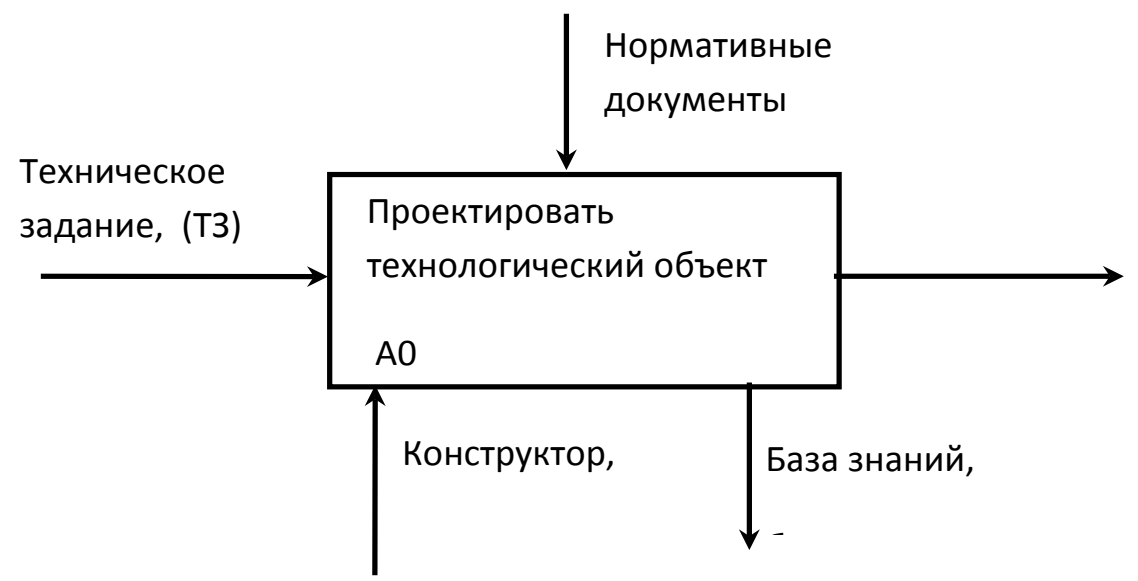

Рисунок 1 - Контекстная диаграмма верхнего уровня АО 
Функцией блока А0 является проектирование технологического объекта. На входе этого блока - техническое задание, на выходе - рабочий проект. Конструктор и технолог являются механизмами. Они являются «лицом, принимающим решение» в вопросах выбора. Для работы системы необходимы различные базы данных и базы знаний.

На рисунке 2 представлена функциональная диаграмма A0, отображающая процесс проектирования технологического оборудования.

Блок A1 «Определение структуры технологического объекта» представлен на рисунке 3. Блок А2 «Технологический расчет» состоит из двух подблоков, представленных на рисунке 4. Блок $A 3$ «Разработка конструкции» представлен на рисунке 5.

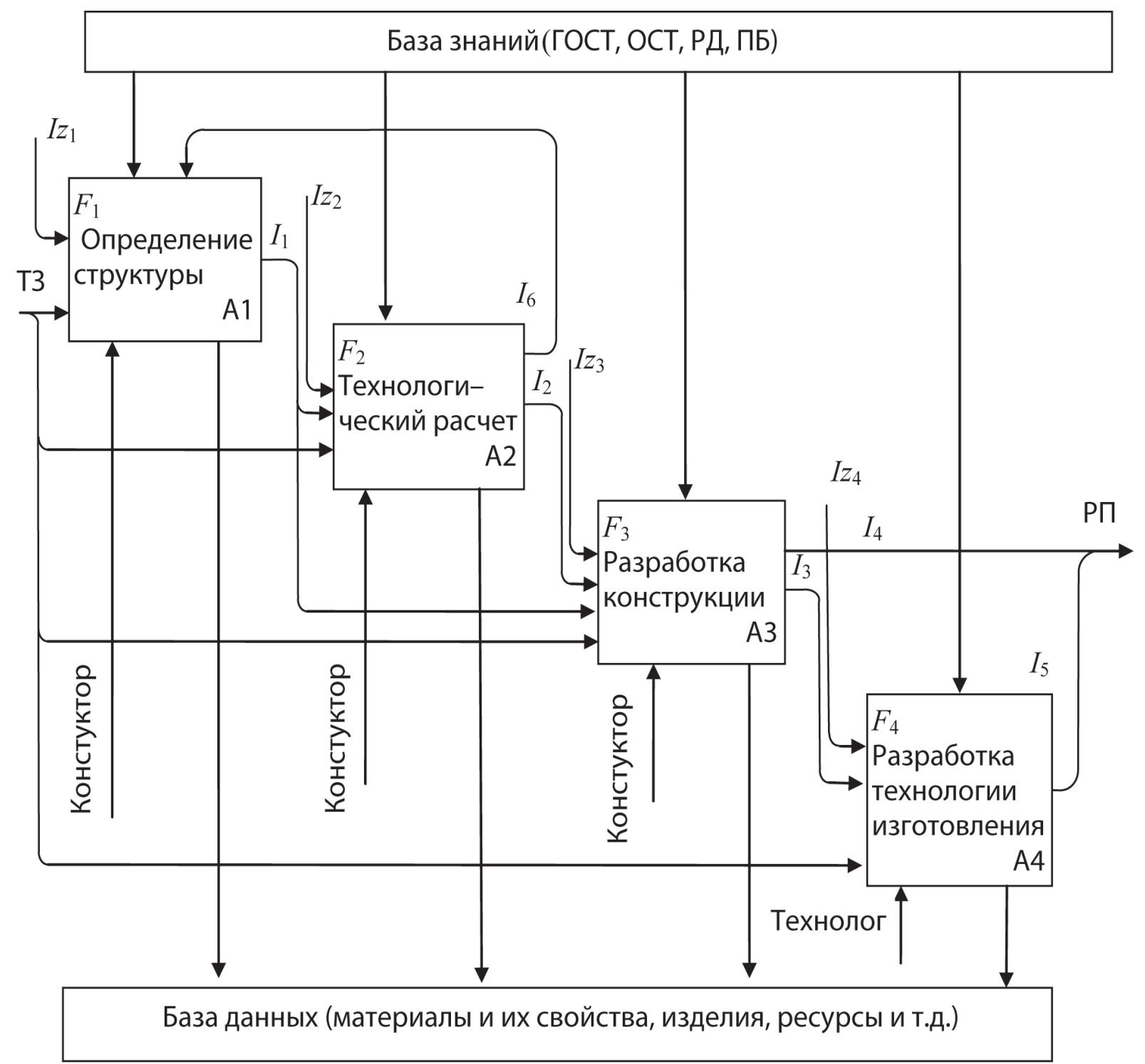

Рисунок 2 - Функциональная диаграмма А0 


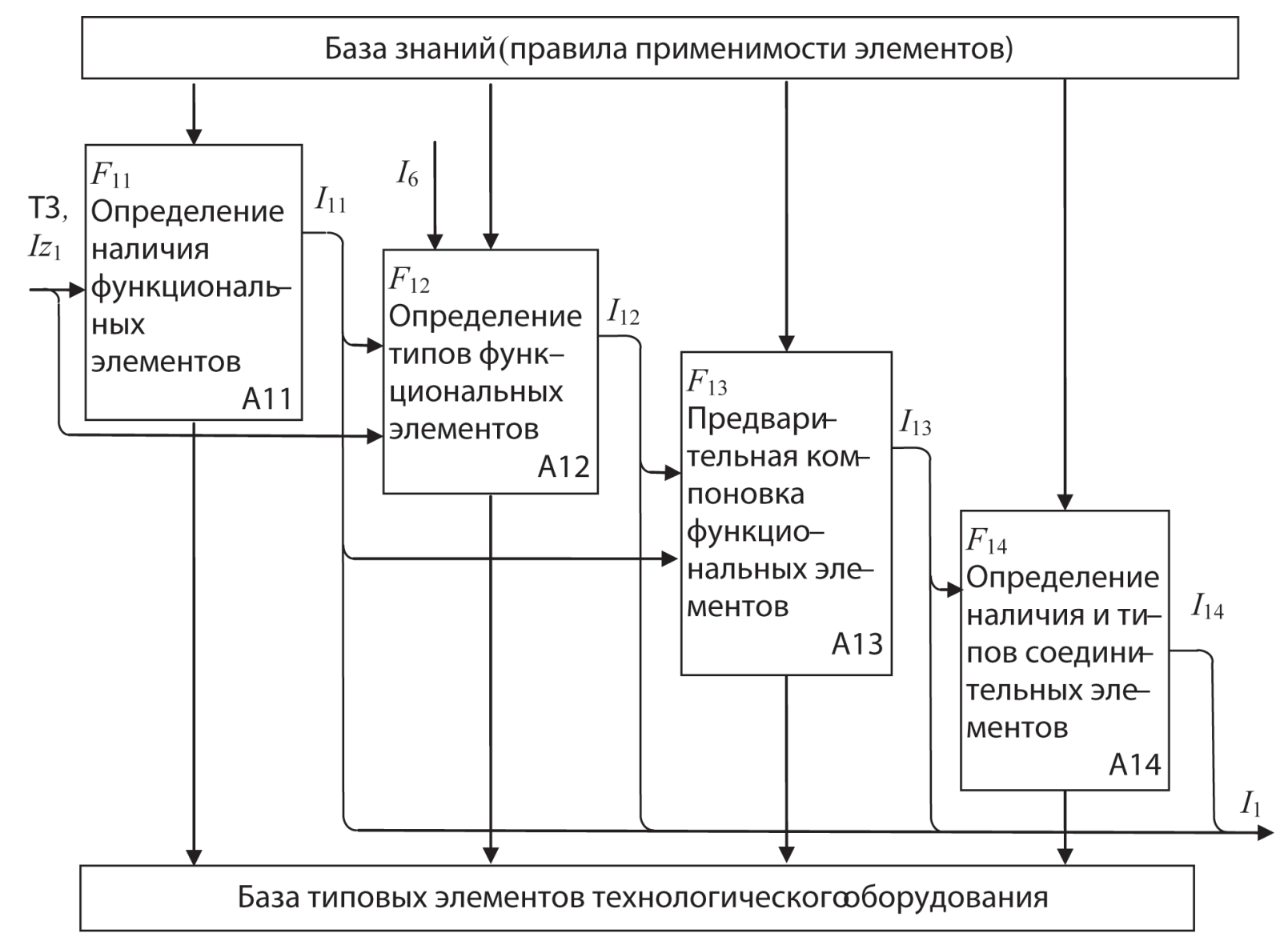

Рисунок 3 - Диаграмма A1 (определение структуры)

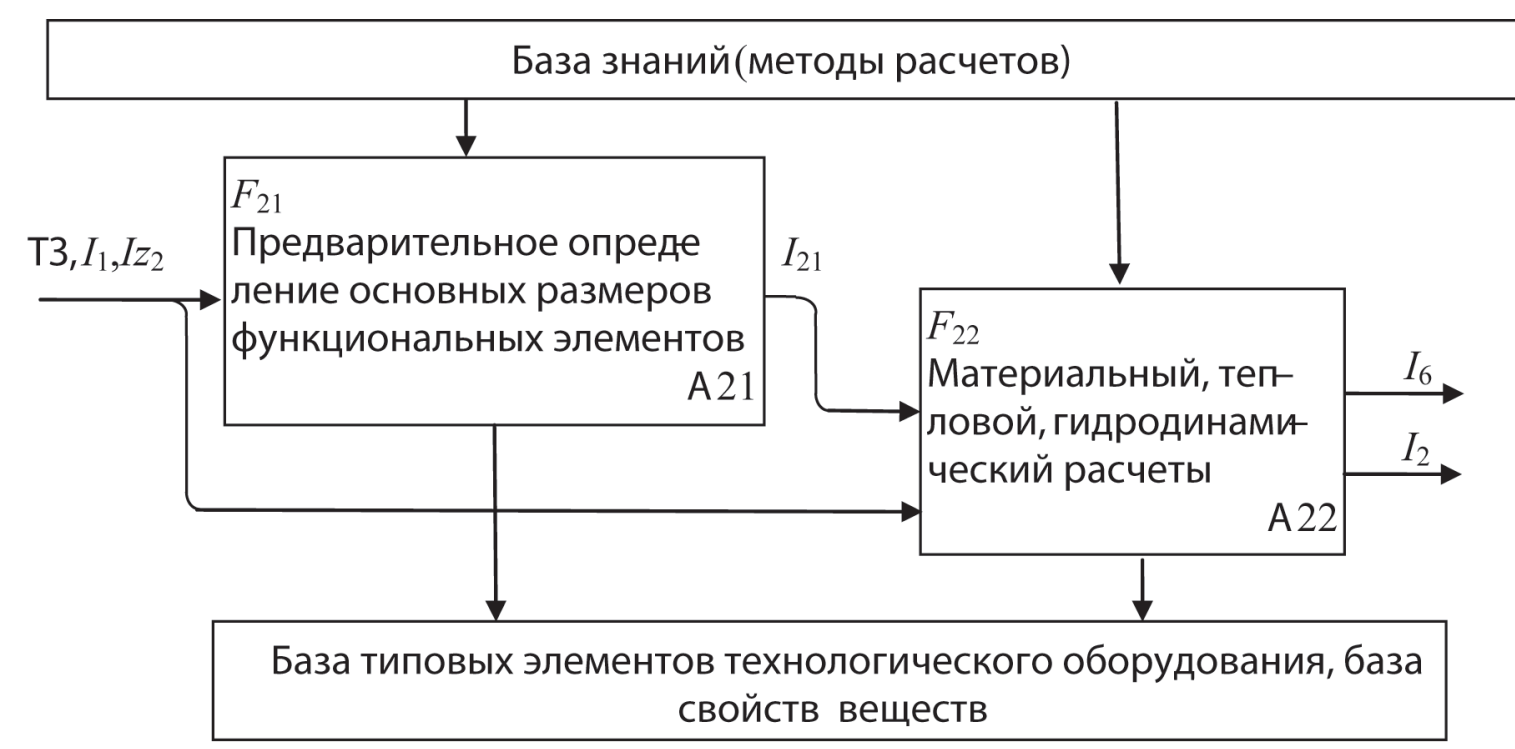

Рисунок 4 - Диаграмма А2 (технологический расчет) 


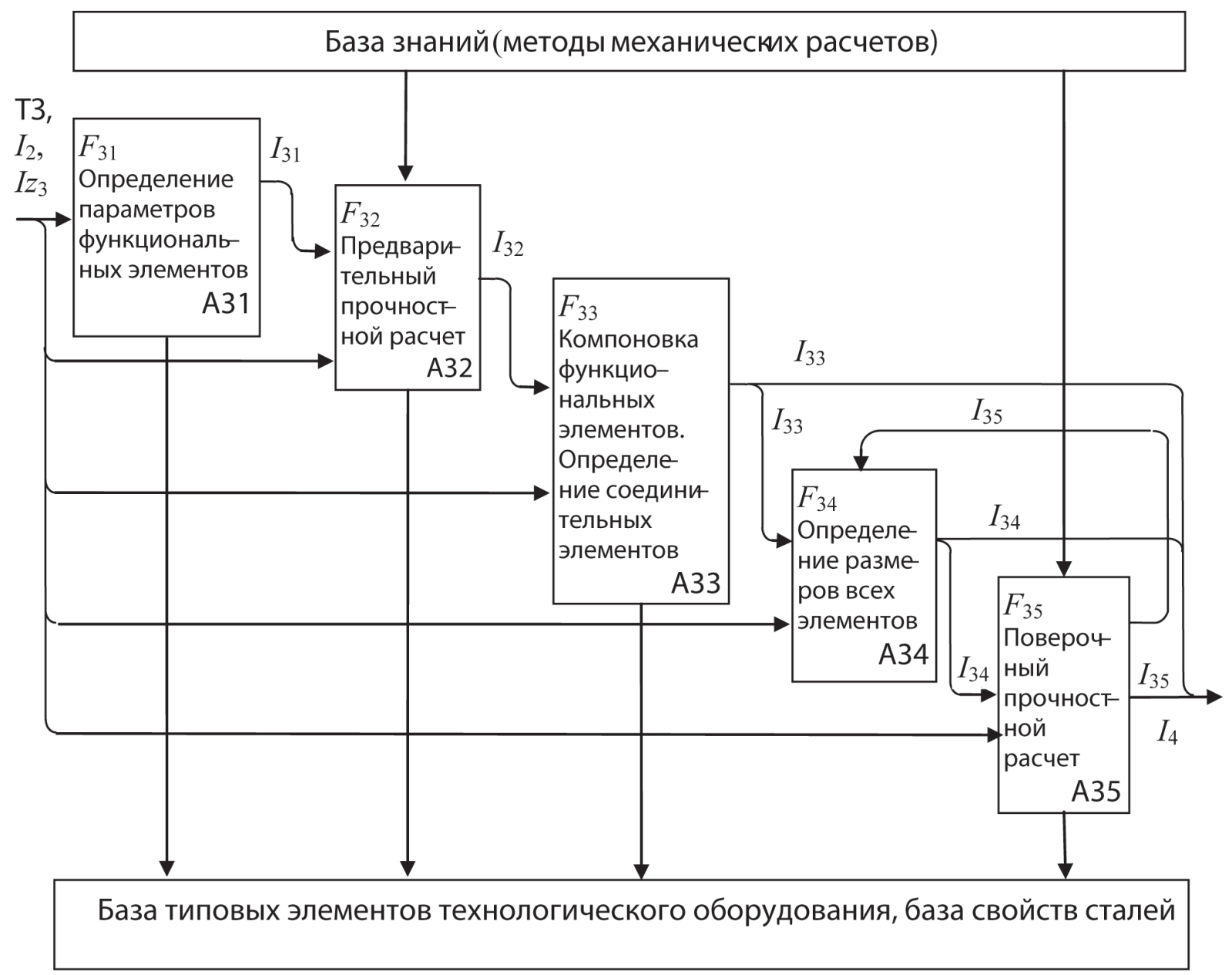

Рисунок 5 - Диаграмма А3 (разработка конструкции)

Информационные потоки, присутствующие при проектировании (рисунки 2, 3, 4, 5) $I_{1}=\left\{I_{11}, I_{12}, I_{13}, I_{14}\right\}$,

где $I_{11}$ - множество данных о наличии функциональных элементов;

$I_{12}$ - множество данных о типах функциональных элементов;

$I_{13}$ - множество данных о взаимном расположении функциональных элементов;

$I_{14}$ - множество данных о наличии и типах соединительных элементов;

$I_{21}$ - предварительные основные размеры функциональных элементов аппарата;

$I_{2}$ - основные размеры и характеристики функциональных элементов аппарата, удовлетворяющие условиям технологического назначения аппарата;

$I_{6}$ - данные, подтверждающие невозможность удовлетворения условиям технологического назначения аппарата при его выбранной структуре;

$I_{7}=\left\{P \Pi_{k}\right\}, k=6$ - технологические расчеты процессов, протекающих в аппарате (тепловые, массообменные, гидродинамические);

$I_{3}=\left\{P \Pi_{k}\right\}, k=1,2,3,4$; 
$I_{31}$ - предварительные основные размеры функциональных элементов аппарата (не определенные ранее в $\left.I_{21}\right)$;

$I_{32}$ - основные размеры функциональных элементов аппарата, определяющие его прочность (такие как толщины элементов корпуса, размеры опор);

$I_{33}$ - уточненные данные о взаимном расположении функциональных элементов, типоразмеры соединительных элементов;

$I_{34}$ - все размеры и другие характеристики всех элементов аппарата, в том числе не определенные ранее в $I_{21}, I_{31}, I_{32}, I_{33}$, а также рабочие чертежи и др. документация;

$I_{35}$ - данные поверочного прочностного расчета;

$I_{4}=\left\{P \Pi_{k}\right\}, k=1,2,3,4,5,7,8$;

$I_{5}=\left\{P \Pi_{k}\right\}, k=9$.

Динамические информационные потоки:

$I z_{1}$ - изменения структуры аппарата (удаления, добавления, изменения типа или взаимного расположения функциональных элементов);

$I z_{2}$ - изменения технологических параметров;

$\mathrm{Iz}_{3}$ - изменения конструкции;

$\mathrm{Iz}_{4}$ - изменения технологии изготовления.

База типовых элементов технологического оборудования должна иметь составляющую (онтологию), которая позволит подбирать типовые элементы в зависимости от условий эксплуатации. Структура такой базы и способы представления знаний достаточно подробно описаны в [8,9].

\section{Процедурная модель процесса проектирования технологического оборудования}

Процедурная модель процесса проектирования $F M$ необходима для разработки на ее основе управляющей программы АИС проектирования технологического оборудования.

Функцией процедурной модели является преобразование информационного потока, определенного техническим заданием ТЗ, в информационный поток рабочего проекта РП

$F M: T 3 \stackrel{M, M^{g}, M^{t}}{\longrightarrow} P \Pi$,

где $M$ - информационно-логическая модель проектируемого объекта;

$M^{g}$ - модели процессов, протекающих в аппарате;

$M^{t}$ - модель технологии изготовления технического объекта.

Процедурная модель $F M$, примененная к $M, M^{g}$ и $M^{t}$, должна позволить на основании технического задания ТЗ получить рабочую документацию РП.

Структура информационно-логической модели технических объектов достаточно подробно описана в [10] и не является предметом настоящей работы.

Модели процессов, протекающих в аппарате, являются предметом специальных исследований. Как правило они базируются на фундаментальных законах тепло- массобмена [11]. 
Модели технологии изготовления технических объектов рассматриваются в работе [12]. Процедурную модель представим в виде системы выражений:

$$
\begin{aligned}
& F M=\left\langle F_{1}, F_{2}, F_{3}, F_{4}\right\rangle, \\
& F_{1}: F a \cup Q_{1} \cup Q_{2} \cup Q_{3} \cup Q_{4} \cup I_{1} \stackrel{M^{S}}{\longrightarrow} I_{1}, \\
& F_{2}: L \cup Q_{1} \cup Q_{2} \cup Q_{4} \cup I_{1} \cup I z_{2} \stackrel{M^{g}, M^{p}}{\longrightarrow}\left(I_{2} \cup I_{7}\right) \vee I_{6}, \\
& F_{3}: L \cup Q_{1} \cup Q_{2} \cup Q_{3} \cup Q_{4} \cup I_{1} \cup I_{2} \cup I_{3} \stackrel{M^{p}, M^{r}}{\longrightarrow} I_{4} \cup I_{3}, \\
& F_{4}: Q_{1} \cup Q_{4} \cup I_{3} \cup I_{4} \stackrel{M^{t}}{\longrightarrow} I_{5},
\end{aligned}
$$

где $F_{1}$ - процедура определения структуры технологического оборудования;

$F_{2}$ - процедура выполнения технологических расчетов оборудования;

$F_{3}$ - процедура разработки конструкции оборудования;

$F_{4}$ - процедура разработки технологии изготовления оборудования;

$M^{S}, M^{p}, M^{r}$ - составляющие информационно-логической модели $M$ проектируемого объекта (модель структуры, модель параметров, модель позиционирования элементов).

Рассмотрим составляющие процедурной модели.

$F_{1}$ - процедура определения структуры оборудования, состоит из следующих составляющих:

$$
\begin{aligned}
& F_{1}=\left\langle F_{11}, F_{12}, F_{13}, F_{14}\right\rangle, \\
& F_{11}: F a \cup Q_{1} \cup Q_{2} \cup Q_{3} \cup Q_{4} \cup I_{1} \stackrel{M^{S}}{\longrightarrow} I_{11}, \\
& F_{12}: F a \cup Q_{1} \cup Q_{2} \cup Q_{3} \cup Q_{4} \cup I_{11} \cup I_{1} \stackrel{M^{S}}{\longrightarrow} I_{12}, \\
& F_{13}: F a \cup Q_{1} \cup Q_{2} \cup Q_{3} \cup Q_{4} \cup I_{12} \cup I_{1} \stackrel{M^{S}}{\longrightarrow} I_{13}, \\
& F_{14}: F a \cup Q_{1} \cup Q_{2} \cup Q_{3} \cup Q_{4} \cup I_{13} \cup I_{1} \stackrel{M^{S} \longrightarrow}{\longrightarrow} I_{14},
\end{aligned}
$$

где $F_{11}$ - процедура определения наличия функциональных элементов оборудования. Так как набор функциональных элементов, которые могут входить в технологический аппарат, известен, нужны правила, определяющие необходимость в наличии каждого из этих элементов. Такие правила могут быть достаточно простыми, так, например, если в T3 в перечне функций оборудования имеется функция «нагреть», то, следовательно, в нем должно присутствовать теплообменное устройство, если других функций не требуется, то это может быть теплообменный аппарат. Так можно определить все основные элементы, входящие в технологический аппарат;

$F_{12}$ - процедура определения типа каждого из функциональных элементов. Здесь правила обычно не являются строго определенными, основываются на накопленном 
в области проектирования опыте, на предпочтениях данного завода изготовителя, на особых требованиях заказчика, то есть ТЗ. Например, известно, что для сыпучих рабочих сред предпочтительный тип днища - коническое, или для вязкой среды предпочтительно перемешивающее устройство с рамной мешалкой;

$F_{13}$ - процедура, выполняющая предварительную компоновку функциональных элементов. Например, определяется местоположение перемешивающего устройства относительно корпуса в емкостном аппарате: сверху, снизу или сбоку, или определяется расположение различных штуцеров и другие;

$F_{14}$ - процедура, определяющая наличие и типы соединительных элементов оборудования. Определяет типы, размеры и другие свойства соединительных элементов, таких как сварные швы, фланцевые, шпоночные, муфтовые соединения и т.п.

$F_{2}$ - процедура выполнения технологических расчетов, состоит из следующих составляющих:

$$
\begin{aligned}
& F_{2}=\left\langle F_{21}, F_{22}\right\rangle, \\
& F_{21}: L \cup Q_{1} \cup Q_{2} \cup Q_{4} \cup I_{1} \cup I_{2} \stackrel{M^{p}}{\longrightarrow} I_{21}, \\
& F_{22}: L \cup Q_{1} \cup Q_{2} \cup Q_{4} \cup I_{11} \cup I_{21} \cup I_{2} \stackrel{M^{g}}{\longrightarrow}\left(I_{2} \cup I_{7}\right) \vee I_{6},
\end{aligned}
$$

где $F_{21}$ - процедура, определяющая предварительно основные, необходимые для проведения технологических расчетов, размеры функциональных элементов оборудования. Основные размеры, необходимые для проведения материального, гидродинамического и теплового расчетов обычно предварительно задаются на основе различных рекомендаций;

$F_{22}$ - процедура, выполняющая материальный, тепловой и гидродинамический расчеты;

При проведении технологических расчетов уточняются основные размеры или изменяются так, чтобы обеспечивались заданные материальная нагрузка, гидродинамический и тепловой режимы в аппарате. При невозможности обеспечить необходимые материальный, гидродинамический и тепловой режимы при выбранных параметрах оборудования, возможно изменение типов составляющих его элементов или типа оборудования.

$F_{3}$ - процедура разработки конструкции оборудования, состоит из следующих составляющих процедур:

$$
\begin{aligned}
& F_{3}=\left\langle F_{31}, F_{32}, F_{33}, F_{34}, F_{35}\right\rangle, \\
& F_{31}: Q_{1} \cup Q_{2} \cup Q_{3} \cup Q_{4} \cup I_{2} \cup I_{3} \stackrel{M^{p}}{\longrightarrow} I_{31}, \\
& F_{32}: Q_{1} \cup Q_{2} \cup Q_{3} \cup Q_{4} \cup I_{2} \cup I_{31} \cup I_{3} \stackrel{M^{p}}{\longrightarrow} I_{32}, \\
& F_{33}: Q_{1} \cup Q_{2} \cup Q_{3} \cup Q_{4} \cup I_{2} \cup I_{32} \cup I_{3} \stackrel{M^{r}}{\longrightarrow} I_{33},
\end{aligned}
$$




$$
\begin{aligned}
& F_{34}: Q_{1} \cup Q_{2} \cup Q_{3} \cup Q_{4} \cup I_{2} \cup I_{33} \cup I_{3} \stackrel{M^{p}}{\longrightarrow} I_{34}, \\
& F_{35}: Q_{1} \cup Q_{2} \cup Q_{3} \cup Q_{4} \cup I_{2} \cup I_{34} \cup I_{3} \stackrel{M^{p}}{\longrightarrow} I_{35},
\end{aligned}
$$

где $F_{31}$ - процедура, определяющая предварительно основные, не определенные ранее в $F_{2}$, размеры функциональных элементов оборудования (например, типоразмер опор, строповых устройств, условный диаметр штуцеров);

$F_{32}$ - процедура, производящая предварительный прочностной расчет. Включает в себя упрощенный проектировочный расчет для определения толщин элементов, нагруженных давлением, или определения толщин по общим рекомендациям;

$F_{33}$ - процедура, выполняющая уточненную компоновку. Определяет точное позиционирование всех элементов относительно друг друга;

$F_{34}$ - процедура, определяющая не определенные ранее параметры элементов оборудования;

$F_{35}$ - процедура, производящая поверочный прочностной расчет.

Поверочный расчет на прочность включает в себя в общем случае следующие прочностные расчеты:

- поверочный расчет на прочность и устойчивость от действия внутреннего и наружного избыточного давления для всех нагруженных элементов оборудования;

- $\quad$ расчет достаточности укрепления отверстий от действия избыточного давления и внешних нагрузок на штуцера;

- поверочный расчет фланцевых соединений оборудования от действия избыточного давления и внешних нагрузок;

- проверку прочности элементов, испытывающих опорные и строповые нагрузки;

- проверку прочности и устойчивости корпуса и опор от действия ветровых и сейсмических нагрузок;

- проверку усталостной прочности элементов, испытывающих циклические нагрузки; - проверку виброустойчивости, жесткости и прочности вращающихся элементов.

В зависимости от специфики работы конкретного аппарата перечень необходимых прочностных расчетов может изменяться.

По результатам проведенных прочностных расчетов возможен возврат к процедуре $F_{34}$ и изменение размеров элементов.

Входными данными для разработки технологии изготовления является конструкторская документация, а именно, сборочные чертежи аппарата и отдельных его узлов и чертежи всех деталей, на которых указаны все необходимые для изготовления и сборки размеры, виды сварки, материал и тип заготовки для деталей.

В таблице 1 обобщены описанные выше состав и функции процедурной модели.

Как было установлено выше, для выполнения функций, определенных процедурной моделью необходимо иметь информационно-логическую модель (ИЛМ) проектируемого технического объекта $M$, модели процессов, протекающих в аппарате $M^{g}$ и модель технологии изготовления технического объекта $M^{t}$. 
Таблица 1 - Процедурная модель процесса проектирования технологического оборудования

\begin{tabular}{|c|c|}
\hline Процедура & Действие \\
\hline$F M$ & $F M: T 3 \stackrel{M, M^{g}, M^{t}}{\longrightarrow} P \Pi$ \\
\hline$F_{1}$ & $F_{1}: F a \cup Q_{1} \cup Q_{2} \cup Q_{3} \cup Q_{4} \cup I_{1} \stackrel{M^{S}}{\longrightarrow} I_{1}$ \\
\hline$F_{11}$ & $F_{11}: F a \cup Q_{1} \cup Q_{2} \cup Q_{3} \cup Q_{4} \cup I z_{1} \stackrel{M^{S}}{\longrightarrow} I_{11}$ \\
\hline$F_{12}$ & $F_{12}: F a \cup Q_{1} \cup Q_{2} \cup Q_{3} \cup Q_{4} \cup I_{11} \cup I_{1} \stackrel{M^{S}}{\longrightarrow} I_{12}$ \\
\hline$F_{13}$ & $F_{13}: F a \cup Q_{1} \cup Q_{2} \cup Q_{3} \cup Q_{4} \cup I_{12} \cup I_{1} \stackrel{M^{S}}{\longrightarrow} I_{13}$ \\
\hline$F_{14}$ & $F_{14}: F a \cup Q_{1} \cup Q_{2} \cup Q_{3} \cup Q_{4} \cup I_{13} \cup I_{1} \stackrel{M^{S}}{\longrightarrow} I_{14}$ \\
\hline$F_{2}$ & $F_{2}: L \cup Q_{1} \cup Q_{2} \cup Q_{4} \cup I_{1} \cup I_{2} \stackrel{M^{g}, M^{p}}{\longrightarrow}\left(I_{2} \cup I_{7}\right) \vee I_{6}$ \\
\hline$F_{21}$ & $F_{21}: L \cup Q_{1} \cup Q_{2} \cup Q_{4} \cup I_{1} \cup I_{2} \stackrel{M^{p}}{\longrightarrow} I_{21}$ \\
\hline$F_{22}$ & $F_{22}: L \cup Q_{1} \cup Q_{2} \cup Q_{4} \cup I_{11} \cup I_{21} \cup I_{2} \stackrel{M^{g}}{\longrightarrow}\left(I_{2} \cup I_{7}\right) \vee I_{6}$ \\
\hline$F_{3}$ & $F_{3}: L \cup Q_{1} \cup Q_{2} \cup Q_{3} \cup Q_{4} \cup I_{1} \cup I_{2} \cup z_{3} \stackrel{M^{p}, M^{r}}{\longrightarrow} I_{4} \cup I_{3}$ \\
\hline$F_{31}$ & $F_{32}: Q_{1} \cup Q_{2} \cup Q_{3} \cup Q_{4} \cup I_{2} \cup I_{31} \cup I_{3} \stackrel{M^{p}}{\longrightarrow} I_{32}$ \\
\hline$F_{32}$ & $F_{32}: Q_{1} \cup Q_{2} \cup Q_{3} \cup Q_{4} \cup I_{2} \cup I_{31} \cup I_{3} \stackrel{M^{p}}{\longrightarrow} I_{32}$ \\
\hline$F_{33}$ & $F_{33}: Q_{1} \cup Q_{2} \cup Q_{3} \cup Q_{4} \cup I_{2} \cup I_{32} \cup I_{3} \stackrel{M^{r}}{\longrightarrow} I_{33}$ \\
\hline$F_{34}$ & $F_{35}: Q_{1} \cup Q_{2} \cup Q_{3} \cup Q_{4} \cup I_{2} \cup I_{34} \cup I_{3} \stackrel{M^{p}}{\longrightarrow} I_{35}$ \\
\hline$F_{35}$ & $F_{35}: Q_{1} \cup Q_{2} \cup Q_{3} \cup Q_{4} \cup I_{2} \cup I_{34} \cup I_{3} \stackrel{M^{p}}{\longrightarrow} I_{35}$ \\
\hline$F_{4}$ & $F_{4}: Q_{1} \cup Q_{4} \cup I_{3} \cup I_{4} \stackrel{M^{t}}{\longrightarrow} I_{5}$ \\
\hline
\end{tabular}




\section{Заключение}

Описанная процедурная модель является математическим обеспечением интеллектуальной автоматизированной системы проектирования технологического оборудования, позволяющей получать проектную документацию с минимальным участием человека. Дальнейшее развитие представленного математического обеспечения заключается в разработке способов представления и обработки всех составляющих процедурной модели в автоматизированной системе.

\section{Библиография :}

1. Мокрозуб В.Г. Методологические основы построения автоматизированной информационной системы проектирования технологического оборудования / В.Г.Мокрозуб, М.П.Мариковская, В.Е.Красильников // Системы управления и информационные технологии. - 2007. - № 1.2(27). - С. 259-262

2. Мокрозуб В.Г., Красильников В.Е., Мариковская М.П. Интеллектуальная автоматизированная информационная система проектирования химического оборудования // Системы управления и информационные технологии. - 2007. - № 4.2 (30). - С. 264-267.

3. Гаврилова Т.А., Муромцев Д.И. Интеллектуальные технологии в менеджменте: инструменты и системы: Учеб. пособие. 2-е изд. / Высшая школа менеджмента СПбГУ. - СПб.: Изд-во "Высшая школа менеджмента"; Издат.дом С.Петерб. гос. ун-та. - 2008. - 488 с.

4. Евгенев Г.Б. Интеллектуальные системы проектирования : учеб. пособие / М. : Изд-во МГтУ им. Н. Э. Баумана. - 2009. - 334с

5. Методология функционального моделирования. Рекомендации по стандартизации Р 50.1.028-2001 Изд-во стандартов. - 2001. - 49с.

6. Кафаров В.В., Ветохин В.Н. Основы автоматизированного проектирования химических производств / М.: Наука. $-1987 .-624$ с.

7. Норенков И.П. Основы автоматизированного проектирования: Учеб. для вузов. 2-е изд., перераб и доп. / М. Изд-во ТГТУ им. Баумана. - 2002. - 336с.

8. Мокрозуб В.Г., Немтинов В.А., Морозов С.В., Коновалова А.С. База данных стандартных и типовыхэлементов технических объектов // Радиотехника. - 2010. - № 12. - С. 29-32.

9. Мокрозуб В.Г. Таксономия в базе данных стандартных элементов технических объектов // Информационные технологии. -2009 . - № 11. - С. 18-22.

10. Мокрозуб В.Г., Немтинов В.А., Егоров С.Я. Информационно-логические модели технических объектов и их представление в информационных системах // Информационные технологии в проектировании и производстве. - 2010. - № 3. С. 68-73.

11. Кафаров В.В., Глебов М.Б. Математическое моделирование основных процессов химических производств / М. Высшая школа. - 1991. - 400с.

12. Павлов B.B. CALS-технологии в машиностроении (математические модели) / М: ИЦ МГТУ Станкин. - 2002. - 328с. 


\section{References:}

1. Mokrozub V.G. Metodologicheskie osnovy postroeniya avtomatizirovannoi informatsionnoi sistemy proektirovaniya tekhnologicheskogo oborudovaniya / V.G.Mokrozub, M.P.Marikovskaya, V.E.Krasil'nikov // Sistemy upravleniya i informatsionnye tekhnologii. - 2007. - № 1.2(27). - S. 259-262

2. Mokrozub V.G., Krasil'nikov V.E., Marikovskaya M.P. Intellektual'naya avtomatizirovannaya informatsionnaya sistema proektirovaniya khimicheskogo oborudovaniya // Sistemy upravleniya i informatsionnye tekhnologii. - 2007. - № 4.2 (30). - S. 264-267.

3. Gavrilova T.A., Muromtsev D.I. Intellektual'nye tekhnologii v menedzhmente: instrumenty i sistemy: Ucheb. posobie. 2-e izd. / Vysshaya shkola menedzhmenta SPbGU. - SPb.: Izd-vo "Vysshaya shkola menedzhmenta"; Izdat.dom S.Peterb. gos. un-ta. -2008 . $-488 \mathrm{~s}$.

4. Evgenev G.B. Intellektual'nye sistemy proektirovaniya : ucheb. posobie / M. : Izd-vo MGTU im. N. E. Baumana. 2009. $-334 \mathrm{~s}$

5. Metodologiya funktsional'nogo modelirovaniya. Rekomendatsii po standartizatsii R 50.1.028-2001 Izd-vo standartov. - 2001. - 49s.

6. Kafarov V.V., Vetokhin V.N. Osnovy avtomatizirovannogo proektirovaniya khimicheskikh proizvodstv / M.: Nauka. 1987. -624 c.

7. Norenkov I.P. Osnovy avtomatizirovannogo proektirovaniya: Ucheb. dlya vuzov. 2-e izd., pererab i dop. / M. Izd-vo TGTU im. Baumana. - 2002. - 336s.

8. Mokrozub V.G., Nemtinov V.A., Morozov S.V., Konovalova A.S. Baza dannykh standartnykh i tipovykh elementov tekhnicheskikh ob"ektov // Radiotekhnika. - 2010. - № 12. - S. 29-32.

9. Mokrozub V.G. Taksonomiya v baze dannykh standartnykh elementov tekhnicheskikh ob"ektov // Informatsionnye tekhnologii. - 2009. - № 11. - S. 18-22.

10. Mokrozub V.G., Nemtinov V.A., Egorov S.Ya. Informatsionno-logicheskie modeli tekhnicheskikh ob"ektov i ikh pred-

11. stavlenie v informatsionnykh sistemakh // Informatsionnye tekhnologii v proektirovanii i proizvodstve. - 2010. - № 3. S. 68-73.

12. Pavlov V.V. CALS-tekhnologii v mashinostroenii (matematicheskie modeli) / M: ITs MGTU Stankin. - 2002. - 328s. 